\title{
Light-shining-through-wall axion detection experiments with a stimulating laser
}

\author{
K. A. Beyer®, ${ }^{1, *}$ G. Marocco®, ${ }^{1, \dagger}$ R. Bingham $\odot,{ }^{2,3}$ and G. Gregori ${ }^{1}$ \\ ${ }^{1}$ Department of Physics, University of Oxford, Parks Road, Oxford OX1 3PU, United Kingdom \\ ${ }^{2}$ Rutherford Appleton Laboratory, Chilton, Didcot OX11 OQX, United Kingdom \\ ${ }^{3}$ Department of Physics, University of Strathclyde, Glasgow G4 ONG, United Kingdom
}

(Received 22 October 2021; accepted 3 February 2022; published 25 February 2022)

\begin{abstract}
The collision of two real photons can result in the emission of axions. We investigate the performance of a modified light-shining-through-wall (LSW) axion search aiming to overcome the large signal suppression for axion masses $m_{a} \geq 1 \mathrm{eV}$. We propose to utilize a third beam to stimulate the reconversion of axions into a measurable signal. We thereby find that with currently available high-power laser facilities we expect bounds at axion masses between $0.5-6 \mathrm{eV}$ reaching $g_{\text {ary }} \geq 10^{-7} \mathrm{GeV}^{-1}$. Combining the use of optical lasers with currently operating $\mathrm{x}$-ray free electron lasers, we extend the mass range to $10-100 \mathrm{eV}$.
\end{abstract}

DOI: 10.1103/PhysRevD.105.035031

\section{INTRODUCTION}

The Standard Model (SM) is one of the biggest achievements of modern particle physics. While successful in predicting any terrestrial experiment, it is known to be incomplete. It falls short of explaining the $C P$ symmetry of the strong sector and fails to provide explanations for the energy density content of the universe. In fact, only around $5 \%$ of the energy density of the universe is in ordinary, baryonic matter, around $26 \%$ is in the form of dark matter, which is not contained in the SM.

One elegant solution to both aforementioned problems makes use of the potential generated by pions after quark confinement. Upon the spontaneous breaking of a new chiral, anomalous $U(1)_{\mathrm{PQ}}$ symmetry, the $C P$ violating vacuum angle of quantum-chromodynamics (QCD) effectively becomes a dynamic field and runs, in the potential generated by the pions, to the $C P$-conserving value $\bar{\theta} \sim 0$. This elegant solution was proposed by Peccei and Quinn in $[1,2]$. Weinberg and Wilczek pointed out that the spontaneous breaking of the new $U(1)_{\mathrm{PQ}}$ leads to the appearance of a pseudo Nambu-Goldstone boson, the QCD axion [3,4]. This new particle is a possible candidate to explain the dark matter content of the universe [5-7].

\footnotetext{
${ }^{*}$ Corresponding author. konstantin.beyer@physics.ox.ac.uk

${ }^{\dagger}$ Corresponding author.

giacomo.marocco@physics.ox.ac.uk
}

Published by the American Physical Society under the terms of the Creative Commons Attribution 4.0 International license. Further distribution of this work must maintain attribution to the author(s) and the published article's title, journal citation, and DOI. Funded by SCOAP ${ }^{3}$.
Generic pseudoscalars also arise abundantly in theory extensions beyond the SM, like in the low energy spectrum of string theory $[8,9]$. In the following we shall mean by the term axion both, the $C P$ restoring $\mathrm{QCD}$ axion and any pseudoscalar particle coupling to electromagnetism with the same 5-dimensional operator

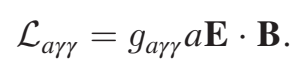

Here, $\mathbf{E}$ and $\mathbf{B}$ are the electric and magnetic field, respectively and $a$ is the axion field. This interaction is polarization dependent and thus perfectly suited for laboratory experiments as the coupling can easily be switched off by a simple change of polarization.

A new, light particle addition to the SM like the axion must be feebly interacting to avoid current detection bounds (see [10]). Such bounds can be broadly classified into three categories, cosmological, astrophysical and laboratory based. The first two types generically outperform laboratory based searches but suffer from varying model dependence like the underlying assumption that the dark matter content of the universe is fully exhausted by the existence of a single axion. For this reason, laboratory based bounds have been called for [11].

Axions are best searched for at the intensity frontier of high power lasers. Axion induced birefringence was searched for by the PVLAS collaboration and its nondetection placed bounds on the axion parameter space [12]. Implementing a traditional Sikivie type light shining through wall (LSW) detector [13], the axion photon coupling $g_{a \gamma \gamma}$ was constrained by multiple groups with the current best bounds set by the QSQAR collaboration [14]. We recently proposed a modified experimental 
approach replacing the static magnetic field of traditional LSW searches by a second laser and thereby avoiding the suppression at large axion masses stemming from the large required momentum transfer [15]. The idea behind the proposal of Ref. [15] is a coherent enhancement of the number of detected photons, $N_{\gamma}$, that is realized via a standing wave setup. However, it can be shown that the setup described in that paper only produces a scaling of $N_{\gamma} \propto|\mathbf{E}|^{2}|\mathbf{B}|^{2} \propto N^{2}$, where $N$ is the number of photons in each of the two lasers used to form the standing wave, instead of the $N^{3}$ scaling as assumed within the quoted bounds of Ref. [15]. Here we aim to clarify that a $N^{3}$ enhancement is still possible if the experimental setup is modified by stimulating the photon regeneration process.

The experimental setup we propose is shown in Fig. 1. The collision of two lasers produces axions, which, due to their weak coupling, traverse a wall blocking the laser light from penetrating into the detector. We propose to replace the static magnetic field detector of traditional LSW searches by an appropriately timed laser beam, thereby avoiding the large suppression for higher axion masses with a larger required momentum transfer for reconversion. The large photon number in high power laser beams stimulates photon production, further enhancing the signal. The latter was described in [16] for an isotropic photon bath and will be applied to a laser beam in Sec. III. Stimulated axion decay can also be used to search for dark matter axions whose decay product produce an echo propagating back to earth when an electromagnetic wave is sent into space $[17,18]$. While we focus on the axion-photon coupling $g_{\text {ary }}$ other schemes investigating the axion's coupling to electrons are investigated in Refs. [19-21].

With the above modification, the proposal has similarities to axion searches via light-by-light scattering, in fact it is an on-shell version of it. The distinct advantages lie in the

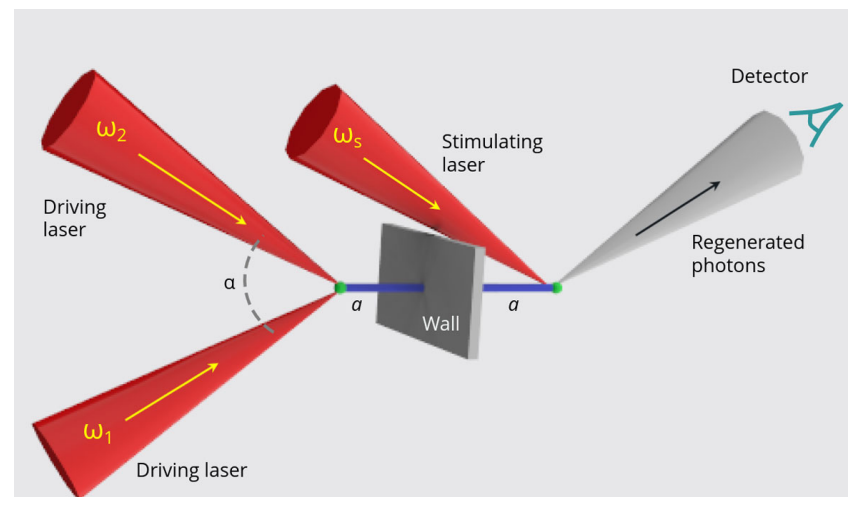

FIG. 1. A diagram of the experimental setup. The collision of two lasers results in the production of any hypothetical axions. Such weakly coupled particles pass through a central wall blocking the laser photons from entering the detector region. An appropriately timed third laser facilitates the reconversion into photons behind the wall. Those reconverted photons are measured with a detector. background suppression due to the spatial and temporal separation of the production and reconversion by the interposing wall and macroscopic distance. Light-by-light scattering for axion detection was investigated in Ref. [22].

The paper is organized as follows, in Sec. II we review the axion production and calculate the axion field we expect for the aforementioned setup. The stimulated reconversion of an axion in a laser beam is then investigated in Sec. III where we find the power in the signal photon field. We finally apply the calculation to our proposed experimental setup and compare the performance to complementary searches in Sec. IV.

\section{PRODUCTION}

The presence of an axion $a$ modifies Maxwell's equations [13] and the resulting wave equations for the fields are

$$
\left(\partial_{t}^{2}-\nabla^{2}\right) \mathbf{E}=g_{a \gamma \gamma}\left[\partial_{t}\left(\mathbf{B} \partial_{t} a-\mathbf{E} \times \nabla a\right)-\nabla[(\nabla a) \cdot \mathbf{B}]\right]
$$

and

$$
\left(\partial_{t}^{2}-\nabla^{2}\right) \mathbf{B}=g_{a \gamma \gamma} \nabla \times\left(\mathbf{E} \times \nabla a-\mathbf{B} \partial_{t} a\right) .
$$

The axion field obeys the Klein-Gordon equation

$$
\left(\partial_{t}^{2}-\nabla^{2}+m_{a}^{2}\right) a=-g_{a \gamma \gamma} \mathbf{E} \cdot \mathbf{B},
$$

where $m_{a}$ is the axion mass. The electric and magnetic fields are produced by two linearly polarized laser beams colliding at an angle $\alpha$. If the pulse length $T$ is much greater than the central frequency $\omega_{j}, j=1,2$, of each laser beam, then the electric and magnetic fields, $\mathbf{E}_{j}$ and $\mathbf{B}_{j}$, respectively, may be treated as a single plane-wave. These may then be decomposed as

$$
\begin{aligned}
& \mathbf{E}_{j}=\frac{1}{2}\left(\mathcal{E}_{j} e^{i \omega_{j} t-i \mathbf{k}_{j} \cdot \mathbf{x}}+\text { c.c. }\right), \\
& \mathbf{B}_{j}=\frac{1}{2}\left(\mathcal{B}_{j} e^{i \omega_{j} t-i \mathbf{k}_{j} \cdot \mathbf{x}}+\text { c.c. }\right),
\end{aligned}
$$

while the axion sourced by these fields is

$$
a=\frac{1}{2}\left[\tilde{a}(\mathbf{x}) e^{i \omega_{a} t}+\text { c.c. }\right],
$$

with

$\left(\omega_{a}^{2}+\nabla^{2}-m_{a}^{2}\right) \tilde{a}(\mathbf{x}) e^{i \omega_{a} t}=\frac{g_{a \gamma \gamma}}{2} \mathcal{F} e^{i\left(\omega_{1}+\omega_{2}\right) t-i\left(\mathbf{k}_{1}+\mathbf{k}_{2}\right) \cdot \mathbf{x}}$

where $\mathcal{F}=\left(\mathcal{E}_{1} \cdot \mathcal{B}_{2}+\mathcal{E}_{2} \cdot \mathcal{B}_{1}\right)$ and $\mathbf{x}$ is the position vector. We will in the following adopt a coordinate system centered on the axion production region. The long laser 
pulse length also fixes the axion energy $\omega_{a}=\omega_{1}+\omega_{2}$ and we define $\mathbf{k}_{a}=\mathbf{k}_{1}+\mathbf{k}_{2}$, whose magnitude is

$$
\left|\mathbf{k}_{a}\right|=\sqrt{\left(\omega_{1}+\omega_{2}\right)^{2}-4 \omega_{1} \omega_{2} \sin ^{2} \frac{\alpha}{2}} \equiv \sqrt{\omega_{a}^{2}-m_{a}^{2}} .
$$

Hence, we see that the collision angle $\alpha$ sets the axion mass the setup tests

$$
m_{a}=\sqrt{4 \omega_{1} \omega_{2} \sin ^{2} \frac{\alpha}{2}}
$$

The fundamental solution to the axion equation is

$$
G(\mathbf{x})=\int \frac{d^{3} k}{(2 \pi)^{3}} \frac{e^{i \mathbf{k} \cdot \mathbf{x}}}{-\omega_{a}^{2}+\mathbf{k}^{2}+m_{a}^{2}}=\frac{e^{-i k_{a}|\mathbf{x}|}}{4 \pi|\mathbf{x}|},
$$

where we neglected the advanced solution and only keep the retarded one. The axion field is then obtained via an integration over the beam overlap region, $V$,

$$
\begin{aligned}
\tilde{a}(\mathbf{x}) & \equiv-\frac{g_{a \gamma \gamma}}{2} \mathcal{F} \int d^{3} y G(\mathbf{x}-\mathbf{y}) e^{-i \mathbf{k}_{a} \cdot \mathbf{y}} \\
& \simeq-\frac{g_{a \gamma \gamma}}{8 \pi} \mathcal{F} \frac{e^{-i k_{a}|\mathbf{x}|}}{|\mathbf{x}|} \int_{V} d^{3} y e^{i k_{a}\left(\hat{x}-\hat{k}_{a}-\frac{\mathbf{y}}{2|\mathbf{x}|}+\frac{\hat{x} \cdot \mathbf{y}}{2 \mid \mathbf{x}} \hat{x}\right) \cdot \mathbf{y}},
\end{aligned}
$$

which applies in the limit where we evaluate the field far from the overlap volume, that is $|\mathbf{x}| \gg|\mathbf{y}|$ and $|\mathbf{x}| \gg \sqrt{k_{a}|\mathbf{y}|^{3}}$.

We can make further simplifications if we consider the direction along the axion momentum $\hat{x}-\hat{k}_{a} \simeq 0$, where $\mathbf{x} /|\mathbf{x}| \equiv \hat{x}$. Treating the overlap $V$ as a cube of sidelength $\ell \ll|\mathbf{x}|$, the integral becomes

$$
\begin{aligned}
& \frac{1}{V} \int_{V} d^{3} y e^{-i k_{a}\left(\frac{\mathbf{y}}{2|\mathbf{x}|}-\frac{\hat{x} \cdot \mathbf{y}}{2|\mathbf{x}|} \hat{x}\right) \cdot \mathbf{y}} \\
& \quad=\left\{\frac{\sqrt{\pi}(1-i)}{\sqrt{\frac{k_{a} \ell^{2}}{|\mathbf{x}|}}} \operatorname{Erf}\left[\frac{(1+i)}{4} \sqrt{\frac{k_{a} \ell^{2}}{|\mathbf{x}|}}\right]\right\}^{2} .
\end{aligned}
$$

As we increase the spot size of the two incoming lasers, therefore increasing $\ell$ and the interaction volume, the axion field amplitude grows linear in volume as long as $\ell^{2}<$ $d / k_{a}$ where we define $d$ the distance to the reconversion region. Increasing the spot size further will only produce a growth linear in $\ell$. In fact the situation is worse because the above scaling is strictly only true when keeping the laser fields $\mathcal{E}_{j}$ constant. In a real laser system of course the energy is constant and therefore the fields scale like $\left|\mathcal{E}_{j}\right|^{2} \propto$ $\ell^{-2}$ resulting in an optimal spot size set by the ratio of separation to axion momentum. In this limit we may approximate (12) by $V$ thus resulting in an axion field given by

$$
\tilde{a}(\mathbf{x}) e^{i \omega_{a} t}=-\frac{g_{a \gamma \gamma}}{8 \pi} V \mathcal{F} e^{i \omega_{a} t} \frac{e^{-i \sqrt{\omega_{a}^{2}-m_{a}^{2}}|\mathbf{x}|}}{|\mathbf{x}|},
$$

at large distances along $\hat{k}_{a}$.

Using this result, we can also calculate the gradient along the observation direction at the position of axion reconversion into photons, that is

$$
\nabla \tilde{a}(d)=-\mathbf{k}_{a} \frac{1+i k_{a} d}{k_{a} d} \tilde{a}(d),
$$

which we will use later.

\section{AXION RECONVERSION}

The produced axions must now be reconverted into photons to leave a detectable signal. Here, we propose to place an opaque wall in the way of the source (drive) lasers, through which, instead, all axions can pass through. These are reconverted by a third laser beam via stimulated axion decay. For simplicity, we choose the stimulating beam to be a copy of either one of the initial beams incident at the same angle on the other side of the wall, see Fig. 1.

The calculation of the signal power proceeds in much the same way as the previous calculation. The only difference is in the perturbation theory of the axion reconversion, in that now we start with an axion field and only one laser, labeled by $s$. We parametrize the signal field as

$$
\mathbf{E}=\frac{1}{2}\left(\tilde{\mathbf{E}}(\mathbf{x}) e^{i \omega t}+\text { c.c. }\right), \quad \mathbf{B}=\frac{1}{2}\left(\tilde{\mathbf{B}}(\mathbf{x}) e^{i \omega t}+\text { c.c. }\right) .
$$

The equations describing the axion-sourced electric field are

$$
\left(-\omega^{2}-\nabla^{2}\right) \tilde{\mathbf{E}}(\mathbf{x}) e^{i \omega t}=-\frac{g_{a \gamma \gamma}}{2}\left\{i\left(\omega_{a}-\omega_{s}\right)\left(\mathcal{E}_{s}^{*} \times \nabla \tilde{a}-i \omega_{a} \mathcal{B}_{s}^{*} \tilde{a}\right) e^{i \mathbf{k}_{s} \cdot \mathbf{x}}+\nabla\left[(\nabla \tilde{a}) \cdot \mathcal{B}_{s}^{*} e^{i \mathbf{k}_{s} \cdot \mathbf{x}}\right]\right\} e^{i\left(\omega_{a}-\omega_{s}\right) t},
$$

and magnetic field

$$
\left(-\omega^{2}-\nabla^{2}\right) \tilde{\mathbf{B}}(\mathbf{x}) e^{i \omega t}=\frac{g_{a \gamma \gamma}}{2} \nabla \times\left(\mathcal{E}_{s}^{*} e^{i \mathbf{k}_{s} \cdot \mathbf{x}} \times \nabla \tilde{a}-i \omega \mathcal{B}_{s}^{*} e^{i \mathbf{k}_{s} \cdot \mathbf{x}} \tilde{a}\right) e^{i\left(\omega_{a}-\omega_{s}\right) t}
$$


The signal photon energy is $\omega=\omega_{a}-\omega_{s}$. Let us start with the electric field and calculate the source from the axion field (13) at the reconversion area which is a large distance $d$ away from the conversion in the direction of the axion momentum. The source density generating the electric field on the right-hand side of (16) is then

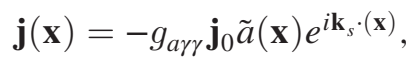

with

$$
\begin{aligned}
\mathbf{j}_{0}= & i \omega\left(\left(\mathcal{E}_{s}^{*} \times \mathbf{k}_{a}\right) \frac{1+i k_{a} d}{k_{a} d}-i \omega_{a} \mathcal{B}_{s}^{*}\right) \\
& +\mathbf{k}_{a}\left(\mathcal{B}_{s}^{*} \cdot \mathbf{k}_{a}\right)\left(\frac{1+i k_{a} d}{k_{a} d}\right)^{2}-i \mathbf{k}_{s}\left(\mathcal{B}_{s}^{*} \cdot \mathbf{k}_{a}\right) \frac{1+i k_{a} d}{k_{a} d} .
\end{aligned}
$$

We find the electric field from the fundamental solution (10) in analogy to before

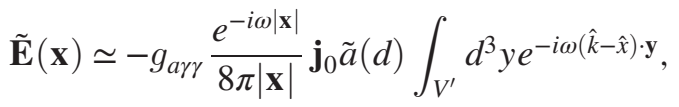

where $V^{\prime}$ is the volume of the reconversion region, and, again, we evaluate the field in the far-field limit $|\mathbf{x}| \gg k \ell^{\prime 2}$ with $\ell^{\prime}$ the sidelength of the reconversion volume, approximated by a cube, and we take the envelope of the source constant over $V^{\prime}$. This time however we wish to maximize the solid angle over which we collect the signal photons, hence we may no longer limit ourselves to a direction parallel $\hat{k}$.

To estimate the signal power we are interested in the intensity in the electromagnetic field a detector at distance $D$ covering a solid angle $d^{2} \Omega$. In the presence of an axion field a nonzero scalar potential $\Phi$ is generated via $\nabla^{2} \Phi=$ $-g_{\text {ary }}(\nabla a) \cdot \mathbf{B}$ resulting in an electric field component parallel to the gradient. Such a field does not propagate in vacuum and will not reach the detector. We may either choose the stimulating laser beam such that $g_{a \gamma \gamma}(\nabla a) \cdot \mathbf{B}=$ 0 and hence restore the gauge freedom to set $\Phi=0$, at least to first order in $g_{a y \gamma}$, or we must limit the detected power to the electric field component orthogonal to the photon momentum $\hat{k}$. The power reaching the detector at distance $D$ is then

$$
\mathcal{P}=\int d \vartheta d \varphi \sin (\vartheta) D^{2}\left|\tilde{\mathbf{E}}(D, \vartheta, \varphi)_{\perp}\right|^{2} \cos ^{2}(\omega t)
$$

The integrand is highly peaked around the photon momentum justifying an integration over the whole sphere as long as our detector is large enough. We thence find, to leading order in $\left(\ell^{\prime} \omega\right)^{-1}$, for the square of the volume integral in Eq. (20):

$$
\frac{64}{\omega^{6}} \int d \vartheta d \varphi \sin (\vartheta) \frac{\sin ^{2}\left\{\frac{\ell^{\prime} \omega}{2}[1-\sin (\theta) \cos (\varphi)]\right\}}{(1-\sin (\theta) \cos (\varphi))^{2}} \frac{\sin ^{2}\left[\frac{\ell^{\prime} \omega}{2} \sin (\theta) \sin (\varphi)\right]}{(\sin (\theta) \sin (\varphi))^{2}} \frac{\sin ^{2}\left[\frac{\ell^{\prime} \omega}{2} \cos (\theta)\right]}{\cos ^{2}(\theta)} \simeq \frac{4 \pi^{2} \ell^{\prime 6}}{\left(\ell^{\prime} \omega\right)^{2}}
$$

where for the exact form of the angular dependence, we assumed the interaction volume to be oriented such that $\hat{k}$ is a unit vector pointing toward one of the faces of the cube $V^{\prime}$. Any other orientation should not change the solution significantly.

We define the geometry of the setup for two laser beams of equal frequency

$$
\begin{aligned}
& \frac{\mathcal{E}_{1}}{\left|\mathcal{E}_{1}\right|}=\left(\begin{array}{l}
0 \\
1 \\
0
\end{array}\right), \quad \frac{\mathcal{B}_{1}}{\left|\mathcal{B}_{1}\right|}=\left(\begin{array}{c}
-\cos \frac{\alpha}{2} \\
0 \\
-\sin \frac{\alpha}{2}
\end{array}\right), \quad \frac{\mathbf{k}_{1}}{\omega_{1}}=\left(\begin{array}{c}
-\sin \frac{\alpha}{2} \\
0 \\
\cos \frac{\alpha}{2}
\end{array}\right) \\
& \frac{\mathcal{B}_{2}}{\left|\mathcal{B}_{2}\right|}=\left(\begin{array}{l}
0 \\
1 \\
0
\end{array}\right), \quad \frac{\mathcal{E}_{2}}{\left|\mathcal{E}_{2}\right|}=\left(\begin{array}{c}
\cos \frac{\alpha}{2} \\
0 \\
-\sin \frac{\alpha}{2}
\end{array}\right), \quad \frac{\mathbf{k}_{2}}{\omega_{2}}=\left(\begin{array}{c}
\sin \frac{\alpha}{2} \\
0 \\
\cos \frac{\alpha}{2}
\end{array}\right)
\end{aligned}
$$

$$
\mathcal{E}_{s}=\mathcal{E}_{2}, \quad \mathcal{B}_{s}=\mathcal{B}_{2}, \quad \mathbf{k}_{s}=\mathbf{k}_{2},
$$

where $\alpha$ is the angle between the two drive beams (see Fig. 1). For a generalization to beams with different frequencies see the Appendix.

The incoming beams are focused such that the beams are cubes of side $\ell$, hence the laser energy contained in the matching interaction volume $E_{j}=\int \mathcal{P}_{j} d \tau=\left|\mathcal{E}_{j}\right|^{2} \ell^{3} / 2$ is simply the laser energy per pulse. This results in the energy of the signal field to be

$$
E=\frac{g_{a \gamma \gamma}^{4}}{64 \pi^{2}} \frac{\ell^{2}}{d^{2}} \omega_{a}^{2} E_{1} E_{2}^{2} \sin ^{4} \frac{\alpha}{2}\left(1-\frac{k_{a}}{\omega_{a}} \cos \frac{\alpha}{2}\right)^{2}
$$

where we set $\ell=\ell^{\prime}$ because the pulse length of the stimulating laser should not be longer than the initial lasers and for simplicity we take it to be a cube again. The dependence on the scattering angle $\alpha$ can be rewritten as an axion mass dependence through (9), resulting, in the case of $\omega_{1}=\omega_{2}$, in 


$$
E=\frac{g_{a \gamma \gamma}^{4}}{64 \pi^{2}} \frac{\ell^{2}}{d^{2}} m_{a}^{2} E_{1} E_{2}^{2}\left(\frac{m_{a}}{\omega_{a}}\right)^{6}
$$

In the general case with different frequency beams the dependence may be more complicated, see the Appendix.

Note, we chose the stimulating beam to be the same as beam 2, such that $\mathcal{B}_{s}^{*} \cdot \mathbf{k}_{a}=0$, simplifying the expression for $\mathbf{j}_{0}$. Performing the full calculation for the other choice of stimulating beam results in the same bounds, thus, justifying this simplifying assumption.

\section{PROJECTED BOUNDS}

To assess the performance of the above proposal we will evaluate the projected bounds utilising the Aton 4 laser at the Extreme Light Infrastructure (ELI) beamlines. This laser system operates at optical frequencies $\omega_{j}=1.55 \mathrm{eV}$ $(j=1,2)$ with $E_{j}=1.5 \mathrm{~kJ}$ energy per pulse and has pulse lengths of $150 \mathrm{fs}$ up to $\tau=1 \mathrm{~ns}$. The optimal pulse duration was discussed earlier and turned out to be $\tau=\sqrt{d / k_{a}}$. The number of signal photons incident on the detector can simply be obtained from the energy equation (26) as $N_{\gamma}=E / \omega$. We will in the following assume single photon counting is possible using a transition edge detector similar to the one designed for the ALPS II experiment $[23,24]$ and exploiting the coincidence timing of signal and incoming lasers to discriminate background. The Aton 4 laser has a repetition rate of $1 \mathrm{~min}^{-1}$ resulting in 1440 shots per day. Assuming a day of data collection per angular step and a required rate of signal photons $R_{\gamma}=1 \mathrm{day}^{-1}$, the projected bounds for this system are

$$
g_{a \gamma \gamma} \geq 3.5 \times 10^{-7} \mathrm{GeV}^{-1}\left(\frac{1.5 \mathrm{~kJ}}{E_{1}}\right)^{\frac{1}{4}}\left(\frac{1.5 \mathrm{~kJ}}{E_{2}}\right)^{\frac{1}{2}}\left(\frac{d}{10 \mathrm{~cm}}\right)^{\frac{1}{4}}\left(\sqrt{1-\left(\frac{m_{a}}{3.08 \mathrm{eV}}\right)^{2}}\right)^{\frac{1}{4}}\left(\frac{3.08 \mathrm{eV}}{m_{a}}\right)^{2}\left(\frac{R_{\gamma}}{\mathrm{day}^{-1}}\right)^{\frac{1}{4}}
$$

where we have taken $\ell=\ell^{\prime}=\tau=\sqrt{d / k_{a}}$ and quote the bounds for the maximal mass, $m_{a} \sim 3.08 \mathrm{eV}$, that can be reached with this setup, obtained by requiring the two beams to be 1 off the counterpropagation direction. The testable parameter space is shown in red in Fig. 2 with the dashed red line the projection obtained assuming $E_{1}=E_{2}=15 \mathrm{~kJ}$. Such increase in laser energy may be within reach by the next generation of high-power laser systems. The lower cutoff in mass assumes a minimal angle of collision $\alpha=1$. In principle, we can extend the exclusion region to lower masses by exploiting the collision of two photons in a converging beam geometry, at arbitrarily small angles similar to what shown in Ref. [25]. However, in that case, the predicted bounds will fall below those already excluded by PVLAS, and they will not probe any new parameter space. Additional increase in the mass range of predicted bounds, also shown in Fig. 2, exploits the use of frequency doubled beams. In estimating these bounds we have assumed a $10 \%$ energy loss for frequency doubling, but such assumption only affects the projected bounds weakly.

To extend the exclusion bounds to even higher axion masses we consider exchanging one of the drive beams with an $\mathrm{x}$-ray free electron laser (XFEL). The European XFEL operates at $\omega_{1}=1 \mathrm{keV}$ with a pulse length $\tau=100 \mathrm{fs}$ and energy per pulse of $E_{1}=0.5 \mathrm{~mJ}$. The shorter pulse length limits the interaction region to a cube of side $\tau$ and the resulting bounds are shown in purple in Fig. 2 for the same distance $d=10 \mathrm{~cm}$. The stimulating laser is a copy of the optical beam to ensure a favorable scaling with the large energy available with such lasers $\left(E_{2}=E_{s}=1.5 \mathrm{~kJ}\right)$. The right region extends the mass range considerably because the frequency of the European XFEL may be tuned up to $\omega=25 \mathrm{keV}$. In principle one can go ahead and exchange

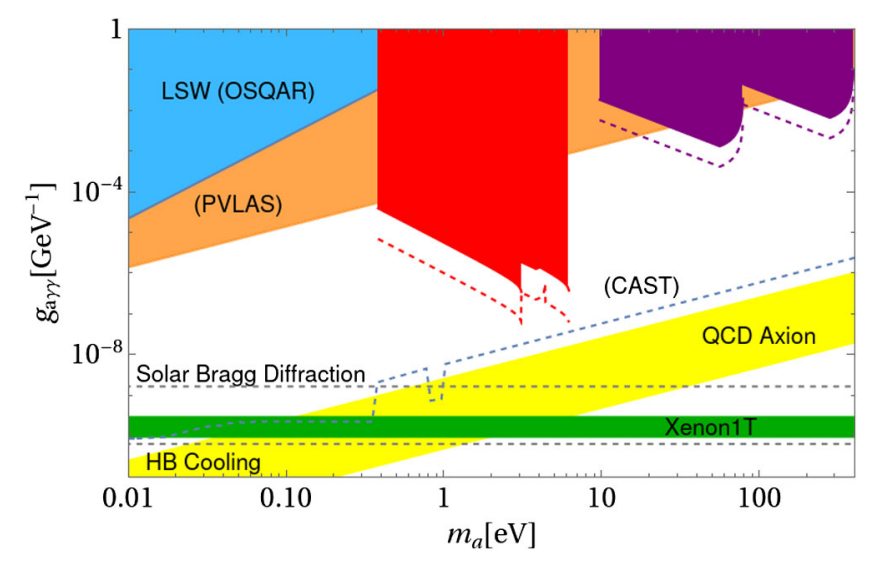

FIG. 2. Exclusion plot for axion parameter space. The light blue region shows existing bounds from the OSQAR experiment [14]; the orange region is excluded by PVLAS [12]; the dashed blue line depicts CAST constraints [26]; the lower horizontal dashed line comes form stellar cooling lifetimes [27] and the upper from solar Bragg diffraction experiments [28]. The green region shows the Xenon1T anomaly interpreted as QCD axion signal [29,30]. The red region on the left indicates the reach of the setup described in the main text using three optical lasers. We included the mass interval which can be probed when considering frequency doubled beams. The dashed red line indicates the improvement for a $15 \mathrm{~kJ}$ laser. The purple region on the right shows the projected bounds for the collision of an optical $15 \mathrm{~kJ}$ laser and an X-FEL like the european X-FEL. The bounds extend from $\omega=1 \mathrm{keV}$, on the left, to $\omega=25 \mathrm{keV}$ on the right and again, the dashed line is a projection to $15 \mathrm{~kJ}$ optical laser energy. The QCD axion region, shown in yellow, indicates particular theoretical predictions for where the axion might be, given dark matter abundances [31]. 
all optical beams for XFEL ones, however, due to the decrease in total power this strategy quickly becomes suboptimal. In drawing the exclusion regions as continuous areas we made the same set of assumptions as was already discussed in Ref. [15]. In a real laser system the spectral width ensures a width in axion masses we test at each angle. We therefore choose the angular step size such that the excluded region is covered continuously. This is possible in $~ 30$ steps if we assume a minimal collision angle and minimal step-size of 1 .

We conclude that the present scheme is capable of producing a competitive $N^{3}$ scaling with the photon number, and it can access an axion parameter space currently unexplored by laboratory experiments. For higher axion masses, we find that the collision of an optical high power pulse with a $\mathrm{x}$-ray free electron laser produces bounds which still test parameter space formerly not reached by laboratory experiments, however the bounds drop off due to the large decrease of photon numbers in the $\mathrm{x}$-ray beam. Future improvement of laser energy may have the potential to reach the QCD band for $\mathrm{eV}$ masses due to the favorable $N^{3}$ dependence of the signal photons.

\section{ACKNOWLEDGMENTS}

We would like to acknowledge our discussions with Kevin Zhou (Stanford) and Antonino Di Piazza (MPIK) that prompted us working on this topic. G. G. acknowledges partial support from STFC (Grant No. ST/T006277/ 1). The research leading to these results has received funding from AWE plc. British Crown Copyright 2021/AWE.

\section{APPENDIX: COUPLING OF DIFFERENT FREQUENCY BEAMS}

With the definition of the geometry (23) we exploited the symmetry between the two beams present in the collision of two identical (up to polarization and propagation direction) optical beams. This allowed for simple expressions denoting the dependence on the scattering geometry. When quoting the bounds achievable by the collision of an optical beam with a X-FEL we must drop this assumption. Fixing the geometry to have the axion propagate again in the $\hat{z}$ direction we find

$$
\begin{gathered}
\frac{\mathbf{k}_{1}}{\left|\mathbf{k}_{1}\right|}=\frac{1}{\sqrt{\omega_{1}^{2}+\omega_{2}^{2}+2 \omega_{1} \omega_{2} \cos \alpha}}\left(\omega_{2} \sqrt{\sin ^{2} \alpha}, 0, \sqrt{\omega_{2}^{2} \cos ^{2} \alpha+\omega_{1}^{2}+2 \omega_{1} \omega_{2} \cos \alpha}\right), \\
\frac{\mathcal{E}_{1}}{\left|\mathcal{E}_{1}\right|}=(0,1,0), \\
\frac{\mathcal{B}_{1}}{\left|\mathcal{B}_{1}\right|}=\frac{-1}{\sqrt{\omega_{1}^{2}+\omega_{2}^{2}+2 \omega_{1} \omega_{2} \cos \alpha}}\left(\sqrt{\omega_{2}^{2} \cos ^{2} \alpha+\omega_{1}^{2}+2 \omega_{1} \omega_{2} \cos \alpha}, 0, \omega_{2} \sqrt{\sin ^{2} \alpha}\right) .
\end{gathered}
$$

And for the second laser

$$
\begin{gathered}
\frac{\mathbf{k}_{2}}{\left|\mathbf{k}_{2}\right|}=\frac{1}{\sqrt{\omega_{1}^{2}+\omega_{2}^{2}+2 \omega_{1} \omega_{2} \cos \alpha}}\left(\omega_{1} \sqrt{\sin ^{2} \alpha}, 0, \sqrt{\omega_{1}^{2} \cos ^{2} \alpha+\omega_{2}^{2}+2 \omega_{1} \omega_{2} \cos \alpha}\right) \\
\frac{\mathcal{E}_{2}}{\left|\mathcal{E}_{2}\right|}=\frac{1}{\sqrt{\omega_{1}^{2}+\omega_{2}^{2}+2 \omega_{1} \omega_{2} \cos \alpha}}\left(\sqrt{\omega_{1}^{2} \cos ^{2} \alpha+\omega_{2}^{2}+2 \omega_{1} \omega_{2} \cos \alpha}, 0,-\omega_{2} \sqrt{\sin ^{2} \alpha}\right) \\
\frac{\mathcal{B}_{2}}{\left|\mathcal{B}_{2}\right|}=(0,1,0)
\end{gathered}
$$

We then evaluate

$$
\mathcal{F}^{2}=\left|\mathcal{E}_{1}\right|^{2}\left|\mathcal{E}_{2}\right|^{2}\left(\frac{\omega_{1} \omega_{2} \sin ^{2} \alpha+2 \omega_{1} \omega_{2} \cos \alpha-\sqrt{\left(\omega_{1} \cos \alpha+\omega_{2}\right)^{2}\left(\omega_{2} \cos \alpha+\omega_{1}\right)^{2}}+\omega_{1}^{2}+\omega_{2}^{2}}{\omega_{1}^{2}+\omega_{2}^{2}+2 \omega_{1} \omega_{2} \cos \alpha}\right)^{2},
$$

and

$$
\left|\mathbf{j}_{0}\right|^{2}=\omega_{a}^{2} \omega_{1}^{2}\left|\mathcal{E}_{2}\right|^{2}\left[\left(1-\frac{k_{a}}{\omega_{a}} \sqrt{\frac{\left(\omega_{1} \cos \alpha+\omega_{2}\right)^{2}}{\omega_{1}^{2}+\omega_{2}^{2}+2 \omega_{1} \omega_{2} \cos \alpha}}\right)^{2}+\frac{k_{a}^{2}}{\omega_{a}^{2}} \frac{\left(\omega_{1} \cos \alpha+\omega_{2}\right)^{2}}{\left(\omega_{1}^{2}+\omega_{2}^{2}+2 \omega_{1} \omega_{2} \cos \alpha\right)} \frac{1}{\left(k_{a} d\right)^{2}}\right],
$$

where again we chose beam 2 to be the stimulating one. This results in energy of the signal field 


$$
E=\frac{g_{a \gamma \gamma}^{4}}{256 \pi^{2}} \frac{\ell^{2}}{d^{2}} \omega_{a}^{2} E_{1} E_{2}^{2} \frac{\mathcal{F}^{2}}{\left|\mathcal{E}_{1}\right|^{2}\left|\mathcal{E}_{2}\right|^{2}} \frac{\left|\mathbf{j}_{0}\right|^{2}}{\omega_{a}^{2} \omega_{1}^{2}\left|\mathcal{E}_{2}\right|^{2}},
$$

from which we may trivially find the bounds on $g_{a \gamma \gamma}$ as indicated by the purple region in Fig. 2 .

[1] R. D. Peccei and Helen R. Quinn, CP Conservation in the Presence of Pseudoparticles, Phys. Rev. Lett. 38, 1440 (1977).

[2] R. D. Peccei and Helen R. Quinn, Constraints imposed by $\mathrm{CP}$ conservation in the presence of pseudoparticles, Phys. Rev. D 16, 1791 (1977).

[3] Steven Weinberg, A New Light Boson?, Phys. Rev. Lett. 40, 223 (1978).

[4] Frank Wilczek, Problem of Strong $P$ and $T$ Invariance in the Presence of Instantons, Phys. Rev. Lett. 40, 279 (1978).

[5] John Preskill, Mark B. Wise, and Frank Wilczek, Cosmology of the invisible axion, Phys. Lett. 120B, 127 (1983).

[6] L. F. Abbott and P. Sikivie, A cosmological bound on the invisible axion, Phys. Lett. 120B, 133 (1983).

[7] Michael Dine and Willy Fischler, The not so harmless axion, Phys. Lett. 120B, 137 (1983).

[8] Edward Witten, Some properties of $\mathrm{O}(32)$ superstrings, Phys. Lett. 149B, 351 (1984).

[9] Asimina Arvanitaki, Savas Dimopoulos, Sergei Dubovsky, Nemanja Kaloper, and John March-Russell, String axiverse, Phys. Rev. D 81, 123530 (2010).

[10] Particle Data Group, Review of particle physics, Prog. Theor. Exp. Phys. 2020, 083C01 (2020).

[11] Joerg Jaeckel, Eduard Masso, Javier Redondo, Andreas Ringwald, and Fuminobu Takahashi, The need for purely laboratory-based axion-like particle searches, Phys. Rev. D 75, 013004 (2007).

[12] Federico Della Valle, Aldo Ejlli, Ugo Gastaldi, Giuseppe Messineo, Edoardo Milotti, Ruggero Pengo, Giuseppe Ruoso, and Guido Zavattini, The PVLAS experiment: Measuring vacuum magnetic birefringence and dichroism with a birefringent FabryPerot cavity, Eur. Phys. J. C 76, 24 (2016).

[13] P. Sikivie, Experimental Tests of the Invisible Axion, Phys. Rev. Lett. 51, 1415 (1983); 52, 695(E) (1984).

[14] R. Ballou et al., New exclusion limits on scalar and pseudoscalar axionlike particles from light shining through a wall, Phys. Rev. D 92, 092002 (2015).

[15] K. A. Beyer, G. Marocco, R. Bingham, and G. Gregori, Axion detection through resonant photon-photon collisions, Phys. Rev. D 101, 095018 (2020).

[16] Andrea Caputo, Marco Regis, Marco Taoso, and Samuel J. Witte, Detecting the stimulated decay of axions at radio frequencies, J. Cosmol. Astropart. Phys. 03 (2019) 027.

[17] Ariel Arza and Pierre Sikivie, Production and Detection of an Axion Dark Matter Echo, Phys. Rev. Lett. 123, 131804 (2019).
[18] Ariel Arza and Elisa Todarello, The axion dark matter echo: A detailed analysis, Phys. Rev. D 105, 023023 (2022).

[19] B. King, Electron-seeded ALP production and ALP decay in an oscillating electromagnetic field, Phys. Lett. B 782, 737 (2018).

[20] Barry M. Dillon and Ben King, ALP production through non-linear Compton scattering in intense fields, Eur. Phys. J. C 78, 775 (2018).

[21] B. M. Dillon and B. King, Light scalars: Coherent nonlinear Thomson scattering and detection, Phys. Rev. D 99, 035048 (2019).

[22] Soroush Shakeri, David J. E. Marsh, and She-Sheng Xue, Light by light scattering as a new probe for axions, arXiv: hep-ph/2002.06123.

[23] Noëmie Bastidon, Dieter Horns, and Axel Lindner, Quantum efficiency characterization and optimization of a tungsten transition-edge sensor for ALPS II, J. Low Temp. Phys. 184, 88 (2016).

[24] Aaron Spector, ALPS II technical overview and status report, arXiv:1611.05863.

[25] Akihide Nobuhiro, Yusuke Hirahara, Kensuke Homma, Yuri Kirita, Takaya Ozaki, Yoshihide Nakamiya, Masaki Hashida, Shunsuke Inoue, and Shuji Sakabe, Extended search for sub-eV axion-like resonances via four-wave mixing with a quasi-parallel laser collider in a high-quality vacuum system, Prog. Theor. Exp. Phys. 2020, 073 C01 (2020).

[26] V. Anastassopoulos, S. Aune, K. Barth, A. Belov, H. Bräuninger, Giovanni Cantatore, J. M. Carmona, J.F. Castel, S. A. Cetin, F. Christensen et al., New cast limit on the axion-photon interaction, Nat. Phys. 13, 584 (2017).

[27] Adrian Ayala, Inma Domínguez, Maurizio Giannotti, Alessandro Mirizzi, and Oscar Straniero, Revisiting the Bound on Axion-Photon Coupling from Globular Clusters, Phys. Rev. Lett. 113, 191302 (2014).

[28] R. Bernabei, P. Belli, R. Cerulli, F. Montecchia, F. Nozzoli, A. Incicchitti, D. Prosperi, C. J. Dai, H. L. He, H. H. Kuang et al., Search for solar axions by Primakoff effect in NaI crystals, Phys. Lett. B 515, 6 (2001).

[29] E. Aprile et al., Excess electronic recoil events in XENON1T, Phys. Rev. D 102, 072004 (2020).

[30] James B. Dent, Bhaskar Dutta, Jayden L. Newstead, and Adrian Thompson, Inverse Primakoff Scattering as a Probe of Solar Axions at Liquid Xenon Direct Detection Experiments, Phys. Rev. Lett. 125, 131805 (2020).

[31] Luca Di Luzio, Federico Mescia, and Enrico Nardi, Redefining the Axion Window, Phys. Rev. Lett. 118, 031801 (2017). 